\title{
PROGRESS ON THE DESIGN OF THE COUPLING COILS FOR MICE AND MUCOOL*
}

\author{
M. A. Green, D. Li, S. P. Virostek, Lawrence Berkeley Laboratory, Berkeley CA 94720, USA \\ L. Wang, H. Wu, L. K. Li, S. Y. Li, F. Y. Xu, X. L. Guo, C. S. Liu, G. Han, X. K. Liu, L. X. Jia \\ Institute of Cryogenics and Superconductivity Technology, HIT, Harbin China.
}

\section{Abstract}

The Muon Ionization Cooling Experiment (MICE) [1] will demonstrate ionization cooling in a short section of a realistic cooling channel using a muon beam at Rutherford Appleton Laboratory (RAL) in the UK. The MICE RF and Coupling Coil (RFCC) Module consists of a superconducting solenoid mounted around four normal conducting 201.25-MHz RF cavities. The coil package that surrounds the RF cavities is to be mounted in a $1.4 \mathrm{~m}$ diameter vacuum vessel. The coupling coil confines the beam in the RFCC module within the radius of the RF cavity beam windows. Each coupling magnet will be powered by a $300 \mathrm{~A}, 10 \mathrm{~V}$ power supply. The maximum design longitudinal force that will be carried by the cold mass support system is $0.5 \mathrm{MN}$. The detailed design and analysis of the coupling magnet has been completed by ICST. The primary magnetic and mechanical design features of the coils are presented in this paper.

\section{INTRODUCTION}

Lawrence Berkeley National Laboratory (LBNL) has been charged with building two coupling magnets for MICE and a single coupling magnet for the MUCOOL experiment at Fermilab. The purpose of the MUCOOL coupling coil is to test a $201.25-\mathrm{MHz}$ RF cavity in a magnetic field that is similar that encountered in MICE. This experiment is critical in determining the acceleration gradients that can be achieved in a magnetic field. The resources needed to build the coupling magnets were in part used to build the two tracker solenoids for MICE, which have to be delivered to RAL in 2008. When the Institute of Cryogenics and Superconductive Technology (ICST), Harbin Institute of Technology (HIT) joined the MICE collaboration in June 2006, it was agreed that they would build the MICE coupling solenoids. As a result, ICST and LBNL are providing three identical coupling coils for the two experiments. The group at LBNL will provide the superconductor, the $4 \mathrm{~K}$ coolers, the HTS leads and a number of other components for the magnets. ICST has done the detailed engineering of the magnets and they will fabricated and test the three magnets in Harbin China. The location where the final magnetic measurements will done has not been determined by the MICE and MUCOOL collaborations

\footnotetext{
*This work was supported by the Lawrence Berkeley Laboratory and the Office of Science, U.S. Department of Energy under Contract No. DE-AC02-05CH11231.
}

\section{LIMITS ON THE MAGNET DESIGN}

The design limits on the MICE coupling magnets come from the vacuum vessel that surrounds the four $\mathrm{RF}$ cavities and the distance between the 201.25-MHz RF cavity couplers [2], [3]. The RF cavities that are used to accelerate muons after they have been cooled using ionization cooling determine the design of the MICE coupling magnet. Since the MUCOOL coupling magnet is to provide field for a $201.25-\mathrm{MHz}$ cavity that is similar to the MICE cavities, it was decided that three coupling solenoid should be identical. This permits ICST to engineer a single magnet design for all three magnets.

The inner radius of the RFCC vacuum vessel under the coupling magnet is $697 \mathrm{~mm}$. As a result, the inner radius of the coupling coil was set to be $750 \mathrm{~mm}$. The $50 \mathrm{~mm}$ space is occupied by the magnet mandrel, the $60 \mathrm{~K}$ shield, the inner vacuum vessel (4 mm thick) and the MLI.

Length of the coupling-magnet superconducting coil is determined by the spacing between the RF cavity couplers. For the two center cavities of MICE, this spacing was set at $517 \mathrm{~mm}$. After some discussion, it was decided that the RF cavity couplers could fit within some circular indents (about $68 \mathrm{~mm}$ inner radius) that surround the couplers. The cryostat length was set at $485 \mathrm{~mm}$. Figure 1 shows the coupling coil mounted on the RFCC module. Figure 2 shows the coupling magnet cryostat and the components within the cryostat vacuum vessel.

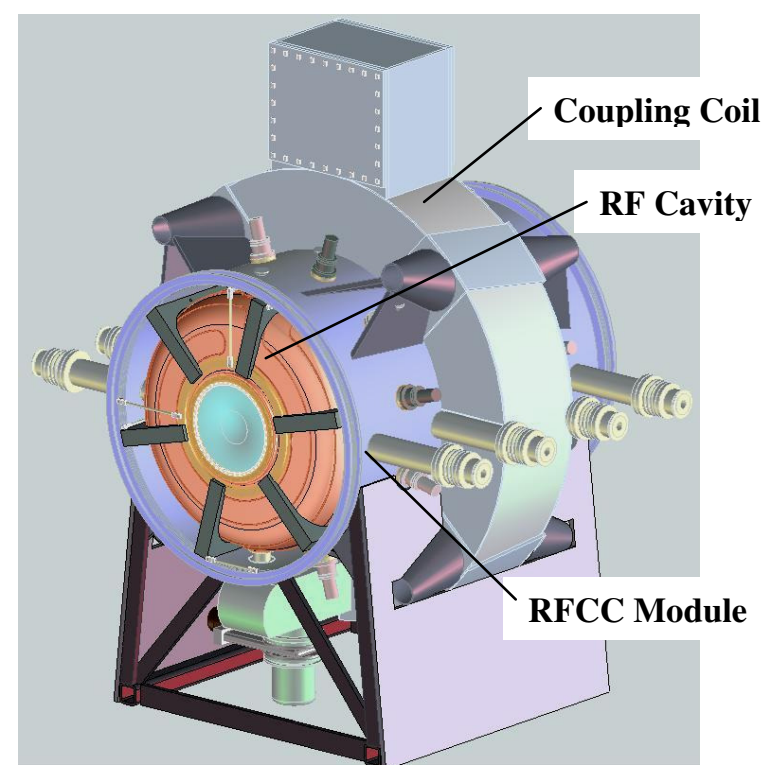

Figure 1. The RFCC Module and Coupling Magnet 


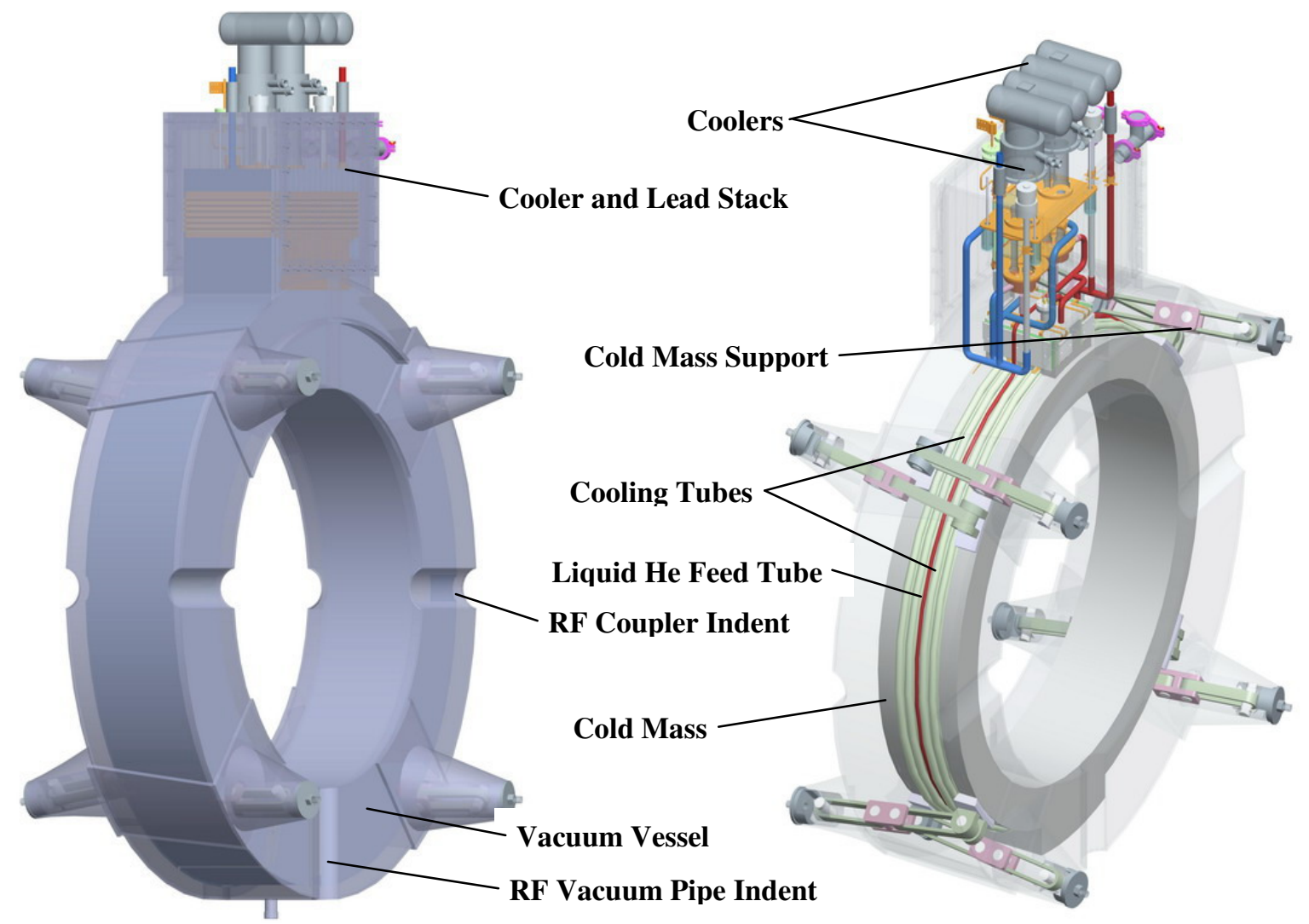

Figure 2. The Coupling Magnet Cryostat Vacuum Vessel (left) and the Coupling Magnet Cold Components (right)

The basic design parameters for the coupling magnet are given in Table 1. The total number of coil turns is 15936, which is more than original design. As result, the coupling coil current is lower than it was for the original design, which increases the temperature margin further. The overall temperature margin is increased by $0.2 \mathrm{~K}$ for the longer coupling magnet design.

Table 1. Coupling Magnet Specifications

\begin{tabular}{lcc}
\hline \multicolumn{1}{c}{ Parameter } & Flip & Non-flip \\
\hline Cryostat Length (mm) & \multicolumn{2}{c}{489} \\
Cryostat Inner Radius (mm) & \multicolumn{2}{c}{700} \\
Cryostat Thickness & \multicolumn{2}{c}{$\sim 350$} \\
Coil Length (mm) & \multicolumn{2}{c}{285} \\
Coil Inner Radius (mm) & \multicolumn{2}{c}{750} \\
Coil Thickness (mm) & \multicolumn{2}{c}{102.5} \\
Number of Layers & \multicolumn{2}{c}{96} \\
No. Turns per Layer & \multicolumn{2}{c}{166} \\
Magnet Self Inductance (H) & \multicolumn{2}{c}{563} \\
Magnet $J\left(A \text { mm }{ }^{-2}\right)^{*}$ & 114.6 & 104.1 \\
Magnet Current (A)* & 210.1 & 190.9 \\
Magnet Stored Energy (MJ)* & 12.4 & 10.3 \\
Peak Induction in Coil (T) & 7.44 & 6.77 \\
Coil Temperature Margin (K) & $\sim 0.8$ & $\sim 1.1$ \\
\hline
\end{tabular}

* Worst case design based on $p=240 \mathrm{MeV} / \mathrm{c}$ and $\beta=420 \mathrm{~mm}$

\section{COUPLING MAGNET CONDUCTOR}

The coupling magnet superconductor will be nearly identical to the conductor that is used in the MICE spectrometer solenoids [4]. The insulated conductor dimensions for the proposed coupling magnet conductor are $1.65 \mathrm{~mm}$ by $1.00 \mathrm{~mm}$. (The bare matrix dimensions are $0.95 \mathrm{~mm}$ by $1.60 \mathrm{~mm}$,) The copper to $\mathrm{S} / \mathrm{C}$ ratio is about 4; the copper RRR is greater than 70; and the conductor twist pitch is $19 \mathrm{~mm}$. The coupling magnet conductor will have 222 filaments that are $41 \mu \mathrm{m}$ in diameter. The conductor specification calls for a conductor critical current that is greater than $760 \mathrm{~A}$ at 5.0 $\mathrm{T}$ and $4.22 \mathrm{~K}$. The cross-section of the proposed conductor for the coupling magnet is shown in Figure 3.

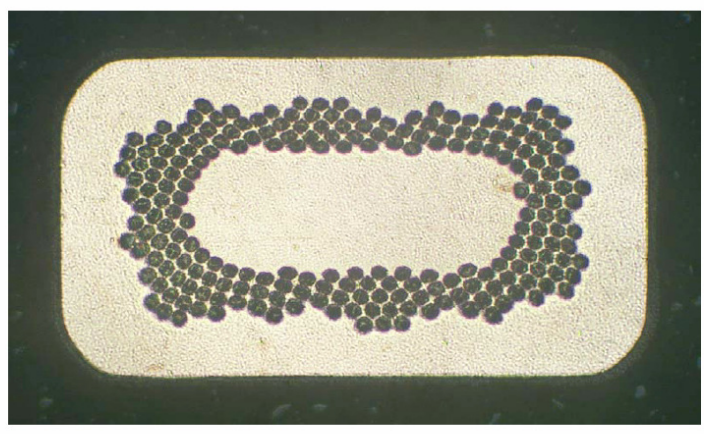

Figure 3. Magnet Conductor Cross-section 


\section{COUPLING MAGNET COOLING}

The MICE and MUCOOL coupling magnet will be cooled by two phase helium in extruded tubes around the outside of the magnet. Condensed liquid helium from the condenser is fed to a liquid helium reservoir at the bottom of the magnet. Two phase helium flows upward through the extruded tubes around the outside of the cold mass. The temperature drop from the high field point in the magnet to the cooling tube is about $0.074 \mathrm{~K}$ when $1.5 \mathrm{~W}$ of heat is put into the magnet from the outside world. The 2-phase helium is collected in a liquid reservoir on the top of the magnet. This reservoir is surrounded by the magnet quench system. The boil off gas flows from the top reservoir to the condensation chamber where the helium is re-condensed. Once the gas is re-condensed, the cold liquid helium flows back down to the bottom of the magnet. This is a typical thermal-siphon cooling loop.

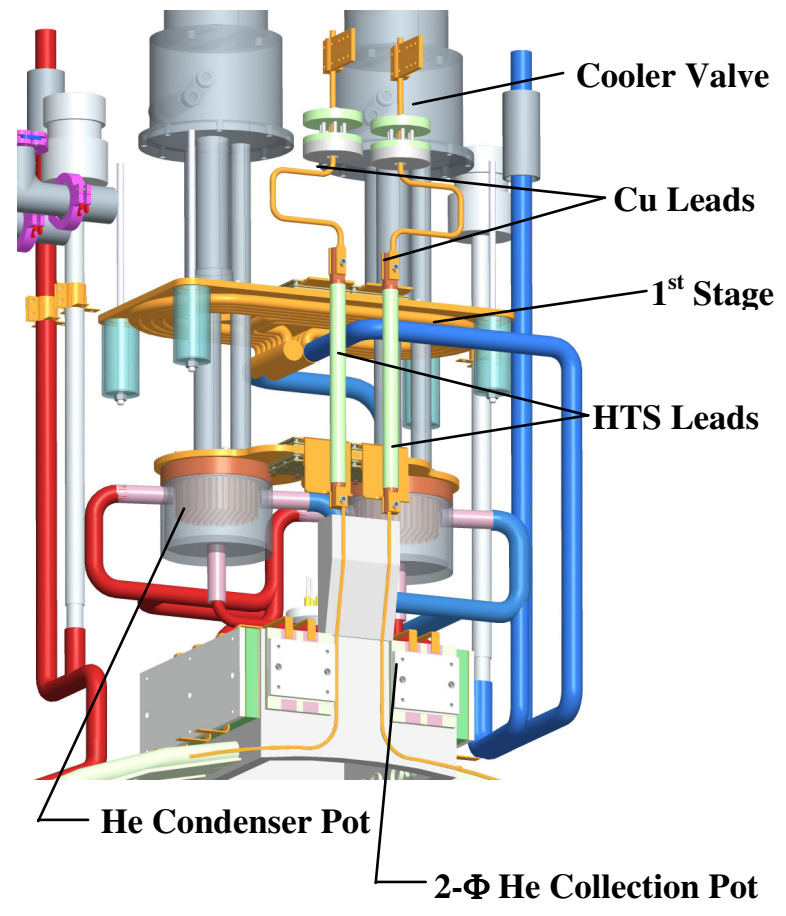

Figure 4. Coupling Magnet Neck Assembly

The coupling magnet neck assembly where two $1.5 \mathrm{~W}$ pulse tube coolers are located is shown in Figure 3. In Figure 4, one can see the cooler valves, the first stage of the coolers, the copper current leads, the HTS current leads, and the helium condensation pot, from which cold liquid helium is sent to the bottom of the magnet bottom. The Cryomech PT415 coolers shown in Figure 3 generate $1.5 \mathrm{~W}$ of cooling at $4.2 \mathrm{~K}$ on the cooler second stage when there is a $42 \mathrm{~W}$ heat load into the first stage.

Table 2 shows the calculated heat loads on the cooler first and second stage temperatures when a single PT-415 pulse tube cooler cools the coupling magnet. Table 2 suggests that the coupling magnet can be kept cold using a single Cryomech PT-415 pulse tube cooler. Since there is uncertainty in the calculated values, the coupling magnet design allows two PT415 coolers to be installed. The installation of a second cooler reduces the first stage temperature to well below $40 \mathrm{~K}$ and as a result reduces the heat load into the cooler second stage.

The AC losses during charging the magnet at a power supply voltage of $10 \mathrm{~V}$ can reach $1.6 \mathrm{~W}$, so even two coolers may not hold the load during the charging of the magnet. The extra helium in the tank above the coils can be boiled away during magnet charging and discharging. This gas can later be re-liquefied by the cooler. The boil off gas generated during magnet charging and discharging is used to cool the region around the upper part of the HTS leads as shown in Figure 4.

Table 2. Projected Coupling Magnet Heat loads

\begin{tabular}{lcc}
\hline \multirow{2}{*}{ Source of Heat Load } & \multicolumn{2}{c}{ Heat Load $(\mathbf{W})$} \\
& $\mathbf{1}^{\text {st }}$ Stage & $\mathbf{2}^{\text {nd }}$ Stage \\
\hline Cold Mass Support & 3.0 & 0.2 \\
MLI Radiation Heat Load & $\sim 8.5$ & $\sim 0.7$ \\
Pipes and Necks & 6.0 & 0.14 \\
Instrumentation Wires & 1.0 & 0.12 \\
Heat Shield Supports & 1.0 & ---- \\
Current Leads & 19.3 & 0.13 \\
Superconducting Joints & --- & 0.01 \\
\hline Total Stage Heat Load $(\mathrm{W})$ & 38.8 & 1.30 \\
Stage Temperature $(\mathrm{K})$ & $\sim 40$ & $\sim 4.05$ \\
\hline
\end{tabular}

\section{CONCLUDING COMMENTS}

The coupling magnets for MICE and MUCOOL will be produced at ICST in collaboration with LBNL. Three identical magnets will be produced (two for MICE and one for MUCOOL). The coupling magnet will be charged to a maximum current of 210 A using a $300 \mathrm{~A}$ power supply. It is hoped the coupling magnet can be kept cold with a single $1.5 \mathrm{~W}$ pulse tube cooler.

\section{REFERENCES}

[1] G. Gregoire, G. Ryckewaert, L. Chevalier, et al, "MICE and International Muon Ionization Cooling Experiment Technical Reference Document," http://hep04.phys.itt.edu/cooldemo

[2] M. A. Green, S. Q. Yang, U. Bravar, et al, "The Mechanical and Thermal Design for the MICE Coupling Solenoid Magnet," IEEE Transactions on Applied Superconductivity 15, No. 2, p 1279, (2005),

[3] M. A. Green, D. Li, S. P. Virostek and H. Witte, "Progress on the Coupling Coil for the MICE Channel," Proceedings of 2005 Particle Accelerator Conference Knoxville TN, p 3468

[4] M. A. Green, C. Y. Chen, T. Juang et al, "Design Parameters for the MICE Tracker Solenoid," to be published in IEEE Transactions on Applied Superconductivity 17, No. 2, (2007). 